\title{
Artificial Coherent States of Light by Multiphoton Interference in a Single-Photon Stream
}

\author{
P. Steindlø, ${ }^{1,{ }^{*}}$ H. Snijders, ${ }^{1}$ G. Westra $\odot,{ }^{1}$ E. Hissink, ${ }^{1}$ K. Iakovlev, ${ }^{1}$ S. Polla $\odot,{ }^{1}$ J. A. Frey, ${ }^{2}$ \\ J. Norman, ${ }^{3}$ A. C. Gossard, ${ }^{3}$ J. E. Bowers, ${ }^{3}$ D. Bouwmeester, ${ }^{1,2}$ and W. Löffler ${ }^{1, \dagger}$ \\ ${ }^{1}$ Huygens-Kamerlingh Onnes Laboratory, Leiden University, P.O. Box 9504, 2300 RA Leiden, Netherlands \\ ${ }^{2}$ Department of Physics, University of California, Santa Barbara, California 93106, USA \\ ${ }^{3}$ Department of Electrical \& Computer Engineering, University of California, Santa Barbara, California 93106, USA
}

(Received 30 October 2020; accepted 5 March 2021; published 6 April 2021)

\begin{abstract}
Coherent optical states consist of a quantum superposition of different photon number (Fock) states, but because they do not form an orthogonal basis, no photon number states can be obtained from it by linear optics. Here we demonstrate the reverse, by manipulating a random continuous single-photon stream using quantum interference in an optical Sagnac loop, we create engineered quantum states of light with tunable photon statistics, including approximate weak coherent states. We demonstrate this experimentally using a true single-photon stream produced by a semiconductor quantum dot in an optical microcavity, and show that we can obtain light with $g^{(2)}(0) \rightarrow 1$ in agreement with our theory, which can only be explained by quantum interference of at least 3 photons. The produced artificial light states are, however, much more complex than coherent states, containing quantum entanglement of photons, making them a resource for multiphoton entanglement.
\end{abstract}

DOI: 10.1103/PhysRevLett.126.143601

Coherent states of light are considered to be the most classical form of light, but expressed in photon number (Fock) space, they consist of a complex superposition of a number of photon number (Fock) states. Because coherent states are nonorthogonal, it is not possible with linearoptical manipulation and superposition of coherent states to obtain pure photon number (Fock) states. The opposite is possible in principle, for instance by attenuating high- $N$ photon number states one could synthesize coherent states. However, high- $N$ Fock states are not readily available, but recently high-quality sources of single-photon $(N=1)$ states became accessible based on optical nonlinearities on the single-photon level. In particular, by using semiconductor quantum dots in optical microcavities [1], singlephoton sources with high brightness, purity, and photon indistinguishability were realized [2-5]. Under loss, in contrast to higher- $N$ Fock states, single-photon streams never lose their quantum character since single photons cannot be split, loss reduces only the brightness. Single photons are an important resource for quantum information applications [6].

In order to synthesize more complex quantum states of light, multiple identical single-photon streams can be combined using beam splitters, where unavoidably quantum interference appears, the well-known Hong-OuMandel (HOM) effect [7]. This effect leads to photon bunching if the incident photons are indistinguishable, therefore enables the production of higher photon number states but only probabilistically. HOM interference is also used for characterization of the photon indistinguishability of single-photon sources [1], which is done mostly in the pulsed regime where detector time resolution is not an issue. The regime of a continuous but random stream of single photons has been explored much less in this aspect, HOM interference with continuous random stream of true single photons has been observed in Refs. [8,9]. The HOM effect can also be used to entangle photons; in combination with single-photon detection and postselection, it also can act as a probabilistic CNOT gate $[6,10,11]$.

Here we make use of HOM interference in a Sagnac-type delay loop with a polarizing beam splitter (Fig. 1), where HOM interference happens at a half-wave plate in polarization space [12]. Similar setups are proposed for boson sampling $[13,14]$ and used for producing linear photonic cluster states [15-17], an emerging resource for universal quantum computation $[6,18,19]$. Since we operate with a random but continuous single-photon stream, the repeated quantum interference and enlargement of the spatiotemporal superposition leads to an infinitely long quantum superposition. By tuning the photon indistinguishability we observe, in agreement with our theoretical model, photon correlations approaching that of coherent light $\left[g^{(2)}(0) \rightarrow 1\right]$, and from our theoretical model, we deduce that the photon number distribution indeed corresponds to coherent light, more precisely weak coherent light with a mean photon number $\bar{n} \approx 0.2$.

Experimentally, as an efficient single-photon source, we use a self-assembled InGaAs/GaAs quantum dot (QD) embedded in a polarization-split micropillar cavity grown by molecular beam epitaxy [5,20]. The QD layer is embedded in a $p-i-n$ junction, separated by a $27 \mathrm{~nm}$-thick tunnel barrier from the electron reservoir, to enable tuning 


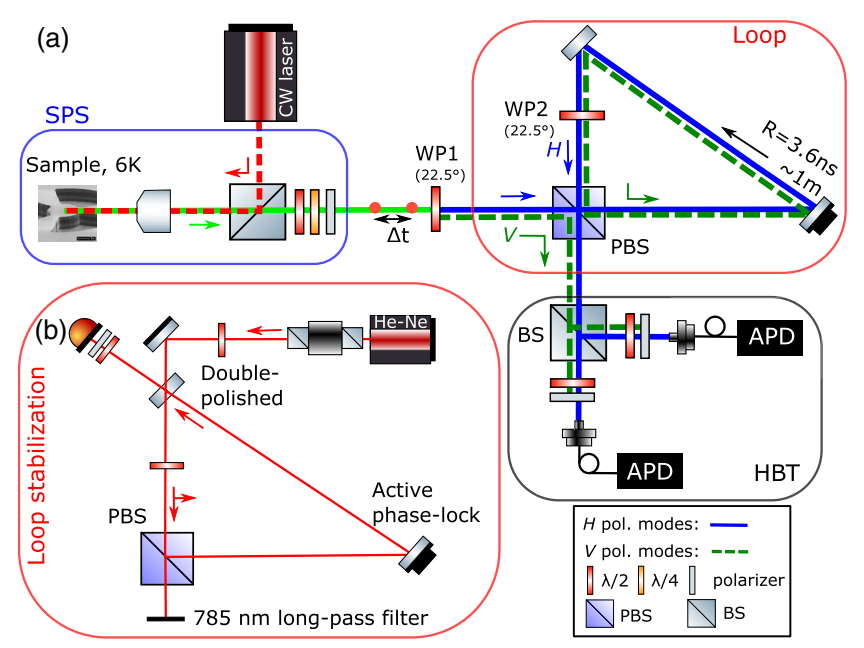

FIG. 1. Experimental setup. (a) Photons from the single-photon source (SPS) are diagonally polarized by WP1 before sent to the loop setup consisting of a polarizing beam splitter and half-wave plate WP2 at $22.5^{\circ}$. Light from the loop setup is analyzed with the polarization-resolved HBT setup. Panel (b) shows the interferometric loop length stabilization.

of the QD resonance around $935 \mathrm{~nm}$ by the quantumconfined Stark effect. The QD transition with a cavityenhanced lifetime of $\tau_{r}=130 \pm 15 \mathrm{ps}$ is resonantly excited with a continuous-wave laser, which is separated by a cross-polarization scheme [20] from the single photons that are collected in a single-mode fiber. This linearly $(H)$ polarized single-photon stream $\Psi_{\text {in }}$ is then brought by WP1 $\left(22.5^{\circ}\right)$ in a superposition of two polarization modes; $\mathrm{H}$ polarized photons enter the $1 \mathrm{~m}$ long free-space delay-loop wherein WP2 $\left(22.5^{\circ}\right)$ brings them again in a superposition, only $\mathrm{H}$-polarized photons are transmitted from the loop towards the detection part. Detection is done with a standard Hanbury Brown-Twiss (HBT) setup with a nonpolarizing beam splitter, after which the photons are coupled into multimode fibers (coupling efficiency $\sim 90 \%$ ) and detected with silicon avalanche photon detectors (APDs, 25\% efficiency) and analyzed with a timecorrelated single-photon counting computer card. With motorized half-wave plates followed by a fixed linear polarizer before each multimode fiber coupler, the setup allows us to distinguish correlations between photons from the loop $\left[g_{H H}^{(2)}(\tau)\right]$, only directly from the source $\left[g_{V V}^{(2)}(\tau)\right]$, and to analyze cross-correlations between photons from the loop and source $g_{V H}^{(2)}(\tau)$. Note that measurement in $V V$ polarization is equivalent to a standard $g^{(2)}(\tau)$ measurement of the single-photon source and can be used to obtain a reference without changing the experimental setup. We have chosen a beam waist of $0.50 \mathrm{~mm}$ inside the loop in order to reduce diffraction loss; the total round-trip transmission $\eta_{L}$ is $\sim 90 \%$. Further, we use active phase stabilization of the loop length by using a mirror on a piezoelectric actuator [Fig. 1(b)] and a frequency-stabilized

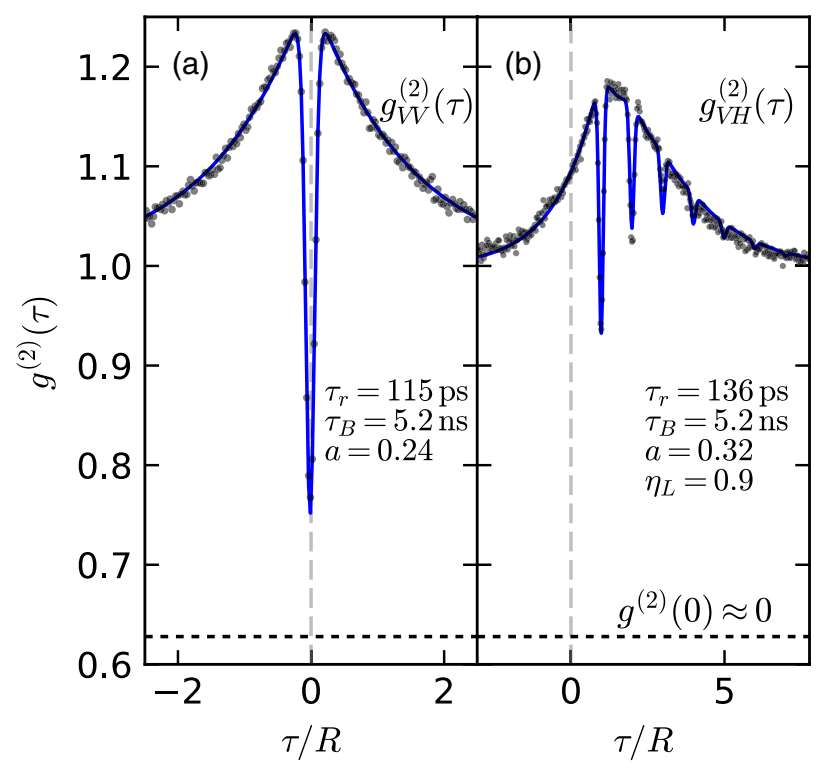

FIG. 2. Characterization of the single-photon source (a) and loop setup (b), experimental data has been accumulated over $3 \mathrm{~h}$; solid lines show the model calculations. In (a) the three-level model is fitted to the experimental $g_{V V}^{(2)}(\tau)$ data to obtain the single-photon source and detector parameters used throughout the Letter. Panel (b) shows $V H$ correlations between photons directly from the source and from the loop, confirming the validity of our model.

He-Ne laser entering the loop through a doubly polished mirror; this is needed because weak pure single-photon states interfere phase sensitively [21].

We operate the QD single-photon source with relatively high excitation power $(\sim 50 \mathrm{nW})$ to obtain a bright singlephoton stream (detected single-photon detection rate of $200 \mathrm{kHz}$ ), with the consequence that unwanted effects produce a broad correlation peak superimposed to $g^{(2)}(\tau)$. In order to correctly take this into account in our model, we first measure in $V V$ detector configuration the source correlations [Fig. 2(a)] and model it using a three-level system [22,23], where $\tau_{B}$ is the lifetime of the additional dark state:

$g_{3 L}^{(2)}(\tau)=1-(1+a) \exp \left(-|\tau| / \tau_{r}\right)+a \exp \left(-|\tau| / \tau_{B}\right)$.

Further, for comparison to experimental results with expected $g^{(2)}(0)$ below 0.1 [5], the theoretical data are convolved with a Gaussian instrument response function (IRF) of our single-photon detectors with FWHM= $0.523 \mathrm{~ns}$ [24], limiting the smallest detectable $g^{(2)}(0) \approx 0.63$. From fitting the model to the experimental data, we obtain a bunching strength $a=0.24 \pm 0.03$ and $\tau_{B}=5.2 \pm 0.3 \mathrm{~ns}$, similar timescales were observed before [25].

To start building up a theoretical model and to characterize the delay loop, we now measure in $V H$ detection configuration the cross-correlation function between 
photons directly from source and photons from the delay loop $g_{V H}^{(2)}(\tau)$, shown in Fig. 2(b). The $V$ detector is connected to the start trigger input of a correlation card and the $H$ detector to the stop channel, therefore the measured correlation $g_{V H}^{(2)}(\tau)$ is as expected asymmetric around $\tau=0$. Considering an $H$-polarized photon entering the loop, WP2 transforms it into an $(1 / \sqrt{2})(|H\rangle+|V\rangle)$ diagonally polarized state. The $H$-polarized part of the state leaves the loop via the polarizing beam splitter, while the $V$ part remains in the loop and is transformed by WP2 into $(1 / \sqrt{2})(|H\rangle-|V\rangle)$, this process is repeating itself infinitely. In the case of a limited amount of photons in welldefined time bins, the output can easily be described, the chance that a photon leaves the loop after $r$ round trips is $\left(\eta_{L} / 2\right)^{r}$ [26]. In our case of a random single-photon stream, the case is more complex as we describe the light stream by correlation functions which we also measure experimentally. In order to predict $g_{V H}^{(2)}(\tau)$ theoretically, we use as an approximation that maximally two photons are in the system, which we prove later to be appropriate here. We obtain for the detected state for two incident photons with delay $\Delta t \neq 0$ (it is a single-photon source) a weighted superposition of single-photon streams shifted by time $r \cdot R$, where $r$ is the round-trip number and $R$ the roundtrip delay (see Supplemental Information [27]):

$\left|\Psi_{V H}\right\rangle=\sum_{\Delta t \neq 0} V^{\dagger}\left[\sqrt{\frac{\eta_{L}}{2}} H_{R+\Delta t}^{\dagger}+\sum_{r \geq 2}\left(-\sqrt{\frac{\eta_{L}}{2}}\right)^{r} H_{r \cdot R+\Delta t}^{\dagger}\right]|0\rangle$.

The state is written in terms of photon creation operators $V_{t}^{\dagger}$ and $H_{t}^{\dagger}$, where the polarization mode is represented by the capital letter, the detection time is given in the subscript. Assuming a source continuously emitting perfect single photons, we can derive from the two-photon state an analytical expression for $g_{V H}^{(2)}(\tau)$ :

$$
g_{V H}^{(2)}(\tau)=1-\sum_{m>0}\left(\frac{\eta_{L}}{2}\right)^{m}\left(1-g_{3 L}^{(2)}(\tau-m \cdot R)\right) .
$$

Here, photons with $\Delta t=z \cdot R, z \in \mathbb{Z}$ are correlated by the loop and create dips in $g_{V H}^{(2)}(\tau)$ for $\tau=m \cdot R$, where $m \in \mathbb{N}$ iterates over round-trips. We observe good agreement between theory and experimental data in Fig. 2(b). Note that also the shifted broad peak originating from strong driving is correctly reproduced.

Finally, we investigate the correlations of photons emerging from the loop by measuring $g_{H H}^{(2)}(\tau)$, shown in Fig. 3. We find that $g_{H H}^{(2)}(\tau=0)$ is now highly sensitive to the indistinguishability or wave function overlap $M$ of consecutive photons produced by the quantum dot, which we can tune experimentally simply by changing the spatial

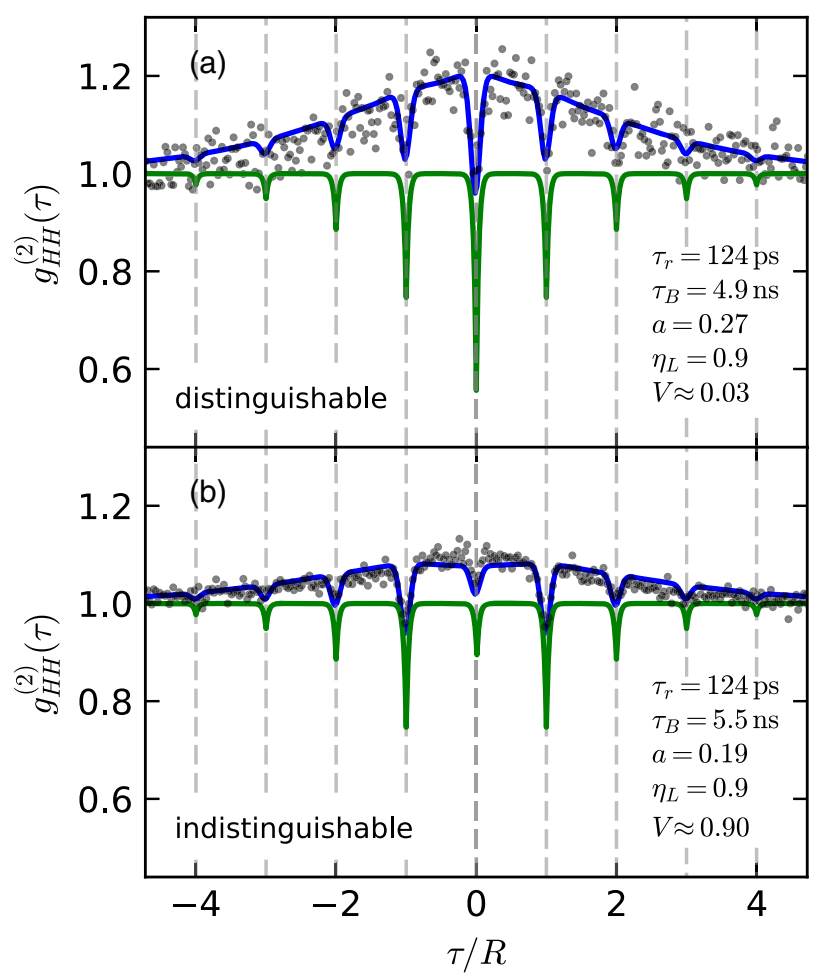

FIG. 3. Photon correlations $g_{H H}^{(2)}(\tau)$ (symbols) for misaligned loop (a, $V \approx 0.03$, distinguishable photons) and aligned loop (b, $V \approx 0.9$, indistinguishable photons) compared to the model predictions (blue curves). Raw coincidence counts corresponding to $g_{H H}^{(2)}(\tau)=1$ were 880 (a) and 9700 (b). The green curves show the model results for the case without spectral diffusion.

alignment of the delay loop. Assuming a perfect singlephoton source, the wave function overlap $M$ is equal to the interferometric visibility $V$, see the Supplemental Material, Sec. C for details [27]. The model for the case of distinguishable photons, shown in Fig. 3(a), can be calculated again in the two-photon picture [27], and we obtain

$$
\begin{aligned}
g_{H H}^{(2)}(\tau)= & 1-\frac{2 \eta_{L}}{4-\eta_{L}^{2}} \sum_{m \in \mathbb{Z} \backslash\{0\}}\left(\frac{\eta_{L}}{2}\right)^{|m|}\left(1-g_{3 L}^{(2)}(\tau-m \cdot R)\right) \\
& -\left(1-g_{H H}^{(2)}(0)\right)\left(1-g_{3 L}^{(2)}(\tau)\right),
\end{aligned}
$$

where the value of $g_{H H}^{(2)}(0)$ has to be calculated using full quantum state propagation which we describe now.

The delay loop leads to quantum interference of photons at different times in the incident single-photon stream, and HOM photon bunching occurring at WP2 produces higher photon number states in a complex quantum superposition. We have developed a computer algorithm that can simulate $g_{H H}^{(2)}(0)$, see the Supplemental Material, Sec. B for details [27]. For the results shown here, we take up to 20 photons or loop iterations into account to approximate the experiment with a continuous photon stream. For completely 


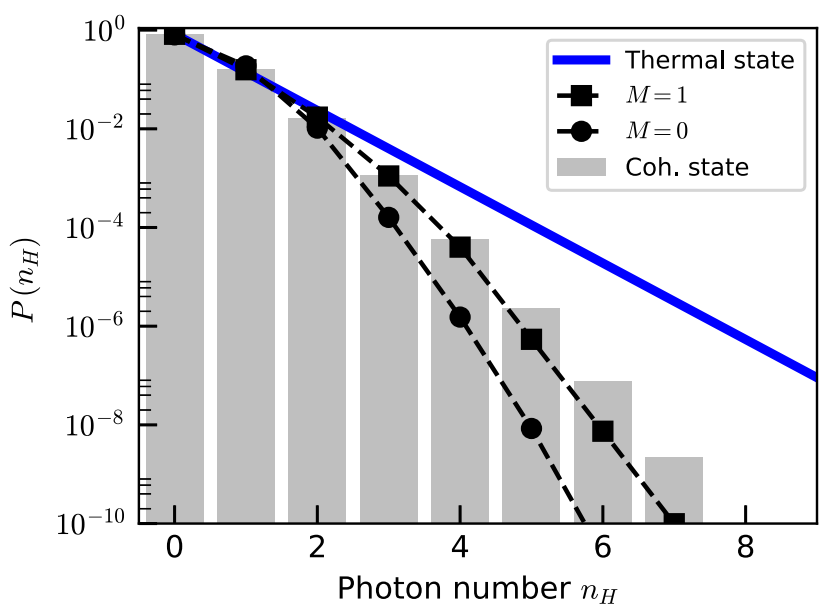

FIG. 4. Comparison of the photon number distribution of a weak coherent state (bars) and thermal state (through blue line) with same mean photon number $\bar{n} \approx 0.2$, to the results from our theoretical model (squares for the case of indistinguishable photons, $M=1$, and circles for the case of distinguishable photons, $M=0$ ). A fixed round-trip loss of 0.1 is included in both cases. The artificial state matches best to the weak coherent light state.

distinguishable photons we obtain $g_{H H}^{(2)}(0)=0.49$ (corrected for dark state dynamics), which agrees well with the experimentally observed correlations in Fig. 3(a).

For the case of indistinguishable photons with maximal wave-function overlap $M \approx 0.9$, we observe in Fig. 3(b) that the dip at $\tau=0$ almost disappears. This is because the (multi-)photon bunching increases the weight of higher photon number states, and, as we show now, produces quasicoherent states of light with $g^{(2)}(0) \approx 1$. Based on our computer simulation, we investigate the photon number distribution $P(n)$, which is shown in Fig. 4. We see very good agreement of the artificial coherent state (indistinguishable photons, $M=1$, experimentally we achieve $M=0.9)$ to an exact weak coherent light state with the same mean photon number $(\bar{n}=0.2)$. In the Supplemental Material [27], Sec. E we show that the artificial coherent state is also very close to being an eigenstate of the annihilation operator, as expected. Now, using the full simulated quantum state, we calculate the quantum fidelity $F$ to the exact coherent state and obtain $1-F \approx 10^{-3}$ for both $M=1$ and $M=0.9$. We also calculate the $l_{1}$ norm of coherence [28] $C_{l_{1}}$, also here the deviation from the exact coherent state is very small, smaller than $10^{-3}$ relatively. From comparison of the density matrices [27], we see that deviations occur mainly in the higher photon number components, those are weak and do not contribute much to the aforementioned measures. These small deviations are also visible in the Wigner function of the artificial coherent state [27].

In the model, we can ignore a round-trip dependent decrease of $M$ due to beam diffraction since the effect is

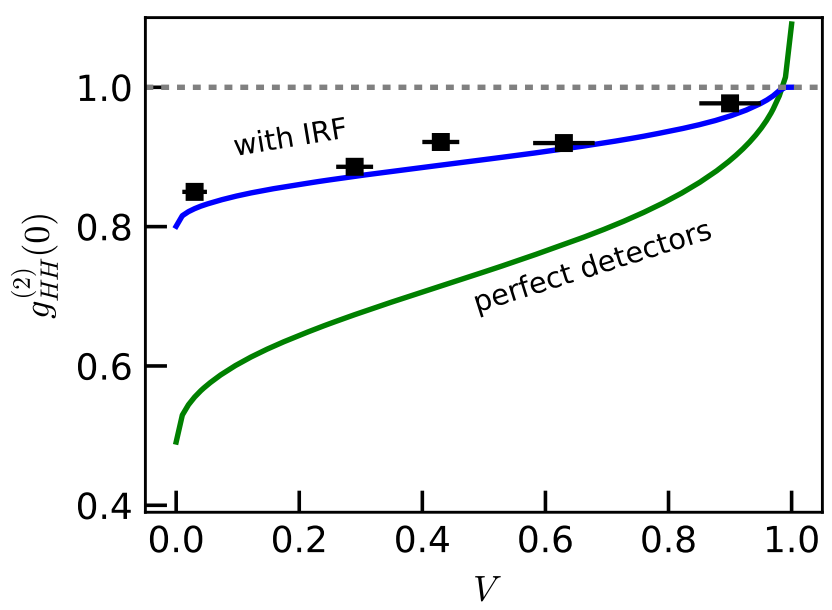

FIG. 5. Experimental $g_{H H}^{(2)}(0)$ (black squares), corrected for dark state dynamics, compared to our theoretical expectations (blue line,convolved with IRF) as a function of the photon indistinguishability or wave-function overlap, expressed by the visibility $V$.Light with coherent photon statistics is obtained for $V \rightarrow 1$. The green line shows the expected results for detectors with perfect timing resolution. Results include a fixed round-trip loss of 0.1 .

only $2 \%$, see Supplemental Material, Sec. B2, and from Fig. 4 we also see why it was justified above to ignore $N>2$ states for prediction of $g_{V H}^{(2)}(\tau)$ and $g_{H H}^{(2)}(\tau \neq 0)$, their contribution is negligible (Supplemental Material, Sec. D [27]). In our experiment, we can also observe the transition to an artificial coherent state by tuning the photon indistinguishability $M$ to intermediate values, which is shown in Fig. 5, again in good agreement with our model. Compared to a weak thermal state of light which can be produced by spontaneous emission of many single-photon emitters coupled to the same cavity mode [29], although having similar $P(n)$ for low $\bar{n}$, as shown in Fig. $4, g^{(2)}(0)$ would show a peak which is not the case here. The simple characterization method based only on two-photon correlations measurement presented here could also be useful for characterization of photonic cluster states demonstrated recently $[16,17]$. In order to determine how many photons are contributing to the quasicoherent states here, by comparing our experimental results to a photon-truncated theoretical model, we see that at least 3 photons are needed to explain our results. We estimate that these three-photon states occur with a rate of about $5 \mathrm{kHz}$ in our experiment [27].

In conclusion, we have shown approximate synthesis of continuous-wave coherent states of light from a quantum dot-based single-photon source, using a simple optical setup with a free-space delay loop. The underlying mechanism is repetitive single-photon addition [30-32] to an ever-growing number-state superposition, and can be tuned by changing photon distinguishability. A difference of the artificial coherent states here to conventional coherent light 
is that the photons of the artificial coherent state are correlated with others separated by multiples of the loop delay, this is typical for systems with time-delayed feedback [33] including lasers [34,35]. This quantum entanglement becomes accessible if an ordered (pulsed) stream of single photons is used, and enables production of linear cluster states, which has been realized recently [16,17], and feed-forward or fast modulators [14,36-38] can be used to produce even more complex quantum states. We want to add that also lasers produce only approximately coherent states with entanglement of the stimulated photons via the gain medium [39-42], which is in practice inaccessible due to the impossibility of monitoring every quantum interaction in the system [43]. From this quantum entanglement arises complexity, therefore we had to use algorithmic modeling in order to produce a theoretical prediction of the output state; this is not surprising because it is known to be computationally hard to calculate quantum interference with many beam splitters (including loop setups such as the one investigated here) and many photons in Fock states, possibly lying beyond the $P$ complexity class $[13,44]$. It would be an interesting goal to develop rigorous entanglement (length) witnesses that can also be applied to continuous and random photon streams such as here, explore possibilities for time-bin encoded tensor networks $[45,46]$ or quantum metrology [47], or to entangle the photons in a $d>1$-dimensional topology [48,49]. A natural question is if other quantum states of light, in particular quadrature squeezed light, can be produced in a similar way, unfortunately, those light states are not resilient against loss compared to coherent states, rendering this far more challenging.

We thank Gerard Nienhuis, Rene Allerstorfer, and Harmen van der Meer for discussions and support, and we acknowledge funding from the EU Horizon 2020 Grant (QLUSTER - 862035), from NWO (08QIP6-2, QUAKE 680.92.18.04), from NWO/OCW as part of the Frontiers of Nanoscience program and the Quantum Software Consortium (024.003.037), and from the National Science Foundation (Q-AMASE-i - DMR-1906325).

*steindl@physics.leidenuniv.nl

${ }^{\dagger}$ loeffler@physics.leidenuniv.nl

[1] C. Santori, D. Fattal, J. Vučković, G. S. Solomon, and Y. Yamamoto, Indistinguishable photons from a single-photon device, Nature (London) 419, 594 (2002).

[2] Y. M. He, Y. He, Y. J. Wei, D. Wu, M. Atatüre, C. Schneider, S. Höfling, M. Kamp, C. Y. Lu, and J. W. Pan, On-demand semiconductor single-photon source with near-unity indistinguishability, Nat. Nanotechnol. 8, 213 (2013).

[3] N. Somaschi, V. Giesz, L. De Santis, J. C. Loredo, M. P. Almeida, G. Hornecker, S. L. Portalupi, T. Grange, C. Antón, J. Demory, C. Gómez, I. Sagnes, N. D. Lanzillotti-Kimura, A. Lemaítre, A. Auffeves, A. G. White,
L. Lanco, and P. Senellart, Near-optimal single-photon sources in the solid state, Nat. Photonics 10, 340 (2016).

[4] X. Ding, Y. He, Z.-C. Duan, N. Gregersen, M.-C. Chen, S. Unsleber, S. Maier, C. Schneider, M. Kamp, S. Höfling, C.-Y. Lu, and J.-W. Pan, On-Demand Single Photons with High Extraction Efficiency and Near-Unity Indistinguishability from a Resonantly Driven Quantum Dot in a Micropillar, Phys. Rev. Lett. 116, 020401 (2016).

[5] H. Snijders, J. A. Frey, J. Norman, V.P. Post, A. C. Gossard, J. E. Bowers, M. P. van Exter, W. Löffler, and D. Bouwmeester, Fiber-coupled cavity-QED source of identical single photons, Phys. Rev. Applied 9, 031002 (2018).

[6] E. Knill, R. Laflamme, and G. J. Milburn, A scheme for efficient quantum computation with linear optics, Nature (London) 409, 46 (2001).

[7] C. K. Hong, Z. Y. Ou, and L. Mandel, Measurement of Subpicosecond Time Intervals between Two Photons by Interference, Phys. Rev. Lett. 59, 2044 (1987).

[8] R. B. Patel, A. J. Bennett, K. Cooper, P. Atkinson, C. A. Nicoll, D. A. Ritchie, and A. J. Shields, Postselective Two-Photon Interference from a Continuous Nonclassical Stream of Photons Emitted by a Quantum Dot, Phys. Rev. Lett. 100, 207405 (2008).

[9] R. Proux, M. Maragkou, E. Baudin, C. Voisin, P. Roussignol, and C. Diederichs, Measuring the Photon Coalescence Time Window in the Continuous-Wave Regime for Resonantly Driven Semiconductor Quantum Dots, Phys. Rev. Lett. 114, 067401 (2015).

[10] M. Larqué, A. Beveratos, and I. Robert-Philip, Entangling single photons on a beamsplitter, Eur. Phys. J. D 47, 119 (2008).

[11] D. Fattal, K. Inoue, J. Vućković, C. Santori, G. S. Solomon, and Y. Yamamoto, Entanglement Formation and Violation of Bell's Inequality with a Semiconductor Single Photon Source, Phys. Rev. Lett. 92, 037903 (2004).

[12] A half-wave plate with its optical axis at $22.5^{\circ}$ acting on the two polarization modes is equivalent to the action of a beam splitter on the two spatial input modes.

[13] K. R. Motes, A. Gilchrist, J. P. Dowling, and P. P. Rohde, Scalable Boson Sampling with Time-Bin Encoding Using a Loop-Based Architecture, Phys. Rev. Lett. 113, 120501 (2014).

[14] Y. He, X. Ding, Z.-E. Su, H.-L. Huang, J. Qin, C. Wang, S. Unsleber, C. Chen, H. Wang, Y.-M. He, X.-L. Wang, W.-J.Zhang, S.-J. Chen, C. Schneider, M. Kamp, L.-X. You, Z. Wang, S. Höfling, C.-Y. Lu, and J.-W. Pan, TimeBin-Encoded Boson Sampling with a Single-Photon Device, Phys. Rev. Lett. 118, 190501 (2017).

[15] E. Megidish, T. Shacham, A. Halevy, L. Dovrat, and H. S. Eisenberg, Resource Efficient Source of Multiphoton Polarization Entanglement, Phys. Rev. Lett. 109, 080504 (2012).

[16] Y. Pilnyak, N. Aharon, D. Istrati, E. Megidish, A. Retzker, and H.S. Eisenberg, Simple source for large linear cluster photonic states, Phys. Rev. A 95, 022304 (2017).

[17] D. Istrati, Y. Pilnyak, J. C. Loredo, C. Antón, N. Somaschi, P. Hilaire, H. Ollivier, M. Esmann, L. Cohen, L. Vidro, C. Millet, A. Lemaître, I. Sagnes, A. Harouri, L. Lanco, P. Senellart, and H.S. Eisenberg, Sequential generation of linear cluster states from a single photon emitter, Nat. Commun. 11, 5501 (2020). 
[18] R. Raussendorf and H. J. Briegel, A One-Way Quantum Computer, Phys. Rev. Lett. 86, 5188 (2001).

[19] P. Walther, K. J. Resch, T. Rudolph, E. Schenck, H. Weinfurter, V. Vedral, M. Aspelmeyer, and A. Zeilinger, Experimental one-way quantum computing, Nature (London) 434, 169 (2005).

[20] H. J. Snijders, D. N. L. Kok, M. F. van de Stolpe, J. A. Frey, J. Norman, A. C. Gossard, J. E. Bowers, M. P. van Exter, D. Bouwmeester, and W. Löffler, Extended polarized semiclassical model for quantum-dot cavity QED and its application to single-photon sources, Phys. Rev. A 101, 053811 (2020).

[21] J. C. Loredo, C. Antón, B. Reznychenko, P. Hilaire, A. Harouri, C. Millet, H. Ollivier, N. Somaschi, L. De Santis, A. Lemaître, T. Sagnes, L. Lanco, A. Auffèves, O. Krebs, and P. Senellart, Generation of non-classical light in a photonnumber superposition, Nat. Photonics 13, 803 (2019).

[22] S. C. Kitson, P. Jonsson, J. G. Rarity, and P. R. Tapster, Intensity fluctuation spectroscopy of small numbers of dye molecules in a microcavity, Phys. Rev. A 58, 620 (1998).

[23] C. Kurtsiefer, S. Mayer, P. Zarda, and H. Weinfurter, Stable Solid-State Source of Single Photons, Phys. Rev. Lett. 85, 290 (2000).

[24] H. Snijders, J. A. Frey, J. Norman, M. P. Bakker, E. C. Langman, A. Gossard, J. E. Bowers, M. P. Van Exter, D. Bouwmeester, and W. Löffler, Purification of a singlephoton nonlinearity, Nat. Commun. 7, 12578 (2016).

[25] M. Davanço, C. S. Hellberg, S. Ates, A. Badolato, and K. Srinivasan, Multiple time scale blinking in InAs quantum dot single-photon sources, Phys. Rev. B 89, 161303(R) (2014).

[26] T. B. Pittman, B. C. Jacobs, and J. D. Franson, Single photons on pseudodemand from stored parametric downconversion, Phys. Rev. A 66, 042303 (2002).

[27] See Supplemental Material at http://link.aps.org/ supplemental/10.1103/PhysRevLett.126.143601 for details of the theoretical derivation of the correlation functions and further characterization of the artificial coherent states.

[28] T. Baumgratz, M. Cramer, and M. B. Plenio, Quantifying Coherence, Phys. Rev. Lett. 113, 140401 (2014).

[29] M. Hennrich, A. Kuhn, and G. Rempe, Transition from Antibunching to Bunching in Cavity QED, Phys. Rev. Lett. 94, 053604 (2005).

[30] S. M. Barnett, G. Ferenczi, C. R. Gilson, and F. C. Speirits, Statistics of photon-subtracted and photon-added states, Phys. Rev. A 98, 013809 (2018).

[31] M. S. Kim, E. Park, P. L. Knight, and H. Jeong, Nonclassicality of a photon-subtracted Gaussian field, Phys. Rev. A 71, 043805 (2005).

[32] B. Marco and Z. Alessandro, Manipulating light states by single-photon addition and subtraction, Prog. Opt. 55, 41 (2010).

[33] A. Pototsky and N. Janson, Correlation theory of delayed feedback in stochastic systems below Andronov-Hopf bifurcation, Phys. Rev. E 76, 056208 (2007).
[34] S. Holzinger, C. Redlich, B. Lingnau, M. Schmidt, M. von Helversen, J. Beyer, C. Schneider, M. Kamp, S. Höfling, K. Lüdge, X. Porte, and S. Reitzenstein, Tailoring the mode-switching dynamics in quantum-dot micropillar lasers via time-delayed optical feedback, Opt. Express 26, 22457 (2018).

[35] T. Wang, Z. L. Deng, J. C. Sun, X. H. Wang, G. P. Puccioni, G. F. Wang, and G. L. Lippi, Photon statistics and dynamics of nanolasers subject to intensity feedback, Phys. Rev. A 101, 023803 (2020).

[36] P. P. Rohde, Simple scheme for universal linear-optics quantum computing with constant experimental complexity using fiber loops, Phys. Rev. A 91, 012306 (2015).

[37] P. Marek, J. Provazník, and R. Filip, Loop-based subtraction of a single photon from a traveling beam of light, Opt. Express 26, 29837 (2018).

[38] V. Švarc, J. Hloušek, M. Nováková, J, Fiurášek, M. Ježek, Feedforward-enhanced Fock state conversion with linear optics, Opt. Express 28, 11634 (2020).

[39] K. Mølmer, Optical coherence: A convenient fiction, Phys. Rev. A 55, 3195 (1997).

[40] S. J. van Enk and C. A. Fuchs, Quantum State of an Ideal Propagating Laser Field, Phys. Rev. Lett. 88, 027902 (2001).

[41] J. Gea-Banacloche, Some implications of the quantum nature of laser fields for quantum computations, Phys. Rev. A 65, 022308 (2002).

[42] D. T. Pegg and J. Jeffers, Quantum nature of laser light, J. Mod. Opt. 52, 1835 (2005).

[43] C. Noh and H. J. Carmichael, Disentanglement of Source and Target and the Laser Quantum State, Phys. Rev. Lett. 100, 120405 (2008).

[44] B. T. Gard, J. P. Olson, R. M. Cross, M. B. Kim, H. Lee, and J.P. Dowling, Inefficiency of classically simulating linear optical quantum computing with Fock-state inputs, Phys. Rev. A 89, 022328 (2014).

[45] M. Lubasch, A. A. Valido, J. J. Renema, W. S. Kolthammer, D. Jaksch, M. S. Kim, I. Walmsley, and R. García-Patrón, Tensor network states in time-bin quantum optics, Phys. Rev. A 97, 062304 (2018).

[46] I. Dhand, M. Engelkemeier, L. Sansoni, S. Barkhofen, C. Silberhorn, and M. B. Plenio, Proposal for Quantum Simulation via All-Optically-Generated Tensor Network States, Phys. Rev. Lett. 120, 130501 (2018).

[47] G. Peniakov, Z.-E. Su, A. Beck, D. Cogan, O. Amar, and D. Gershoni, Towards supersensitive optical phase measurement using a deterministic source of entangled multiphoton states, Phys. Rev. B 101, 245406 (2020).

[48] W. Asavanant, Y. Shiozawa, S. Yokoyama, B. Charoensombutamon, H. Emura, R. N. Alexander, S. Takeda, J. ichi Yoshikawa, N. C. Menicucci, H. Yonezawa, and A. Furusawa, Generation of time-domain-multiplexed twodimensional cluster state, Science 366, 373 (2019).

[49] M. V. Larsen, X. Guo, C. R. Breum, J. S. NeergaardNielsen, and U. L. Andersen, Deterministic generation of a two-dimensional cluster state, Science 366, 369 (2019). 